\title{
SCIENTIFIC REPORTS

\section{Inner histopathologic changes and disproportionate zone volumes in foetal growth plates following gestational hypoglycaemia in rats}

Vivi F. H. Jensen ${ }^{1,2,3^{*}}$, Anne-Marie Mølck ${ }^{1}$, Ingrid B. Bøgh ${ }^{1}$, Jette Nowak ${ }^{1}$, Birgitte M. Viuff ${ }^{1}$, Charlotte L. M. Rasmussen ${ }^{2}$, Louise Pedersen ${ }^{2}$, Johannes J. Fels ${ }^{4}$, Suzi H. Madsen ${ }^{4}$, Fiona E. McGuigan ${ }^{3}$, Pernille Tveden-Nyborg ${ }^{2}$, Jens Lykkesfeldt ${ }^{2}$ \& Kristina E. Akesson ${ }^{3}$

Maternal hypoglycaemia throughout gestation until gestation day (GD)20 delays foetal growth and skeletal development. While partially prevented by return to normoglycaemia after completed organogenesis (GD17), underlying mechanisms are not fully understood. Here, we investigated the pathogenesis of these changes and significance of maternal hypoglycaemia extending beyond organogenesis in non-diabetic rats. Pregnant rats received insulin-infusion until GD20 or GD17, with sacrifice on GD20. Hypoglycaemia throughout gestation increased maternal corticosterone levels, which correlated with foetal levels. Growth plates displayed central histopathologic changes comprising disrupted cellular organisation, hypertrophic chondrocytes, and decreased cellular density; expression of pro-angiogenic factors, HIF-1 $\alpha$ and VEGF-A increased in surrounding areas. Disproportionately decreased growth plate zone volumes and lower expression of the structural protein MATN-3 were seen, while bone ossification parameters were normal. Ending maternal/foetal hypoglycaemia on GD17 reduced incidence and severity of histopathologic changes and with normal growth plate volume. Compromised foetal skeletal development following maternal hypoglycaemia throughout gestation is hypothesised to result from corticosterone-induced hypoxia in growth plates, where hypoxia disrupts chondrocyte maturation and growth plate structure and volume, decreasing long bone growth. Maternal/foetal hypoglycaemia lasting only until GD17 attenuated these changes, suggesting a pivotal role of glucose in growth plate development.

It has previously been established that maternal hypoglycaemia may cause foetal growth restriction and in rodents, even short-term, maternal hypoglycaemia may disturb foetal skeletal development, reflected in decreased ossification and bone malformations ${ }^{1-4}$. The underlying mechanisms are, however, not clear; especially an understanding of the critical time windows of maternal hypoglycaemia and its duration to allow for normal versus abnormal foetal development is still lacking. We therefore established a model in non-diabetic rats to study the effect of sustained insulin-induced hypoglycaemia on foetal skeletal development ${ }^{5}$. Previously we showed that continuous maternal hypoglycaemia throughout gestation resulted in skeletal malformations, as well as decreased skeletal growth and bone mineral density ${ }^{5}$. In comparison hypoglycaemia lasting only until GD17, when organogenesis is completed, followed by normoglycemia allowed for near-normal development, emphasising that sufficient glucose supply late in gestation is crucial $^{6}$.

To elucidate the principal mechanisms underlying the observed foetal skeletal defects, we have taken a wider approach to assess histological changes and to identify potentially regulatory pathways for bone growth and calcification. We hypothesised that hypoglycaemia disrupts regulation of the skeletal micro-environment by altering growth plate structure, as well as gene expression, and hormones essential for normal development of growth

${ }^{1}$ Novo Nordisk A/S, Department of Toxicology, Safety Pharmacology and Pathology, Maaloev, Denmark. ${ }^{2}$ University of Copenhagen, Department of Veterinary and Animal Sciences, Section for Experimental Animal Models, Copenhagen, Denmark. ' ${ }^{2}$ und University, Department of Clinical Sciences Malmö and Skåne University Hospital, Department of Orthopedics, Malmö, Sweden. ${ }^{4}$ Novo Nordisk A/S, Department of Research Bioanalysis, Maaloev, Denmark.*email: vfhj@novonordisk.com 


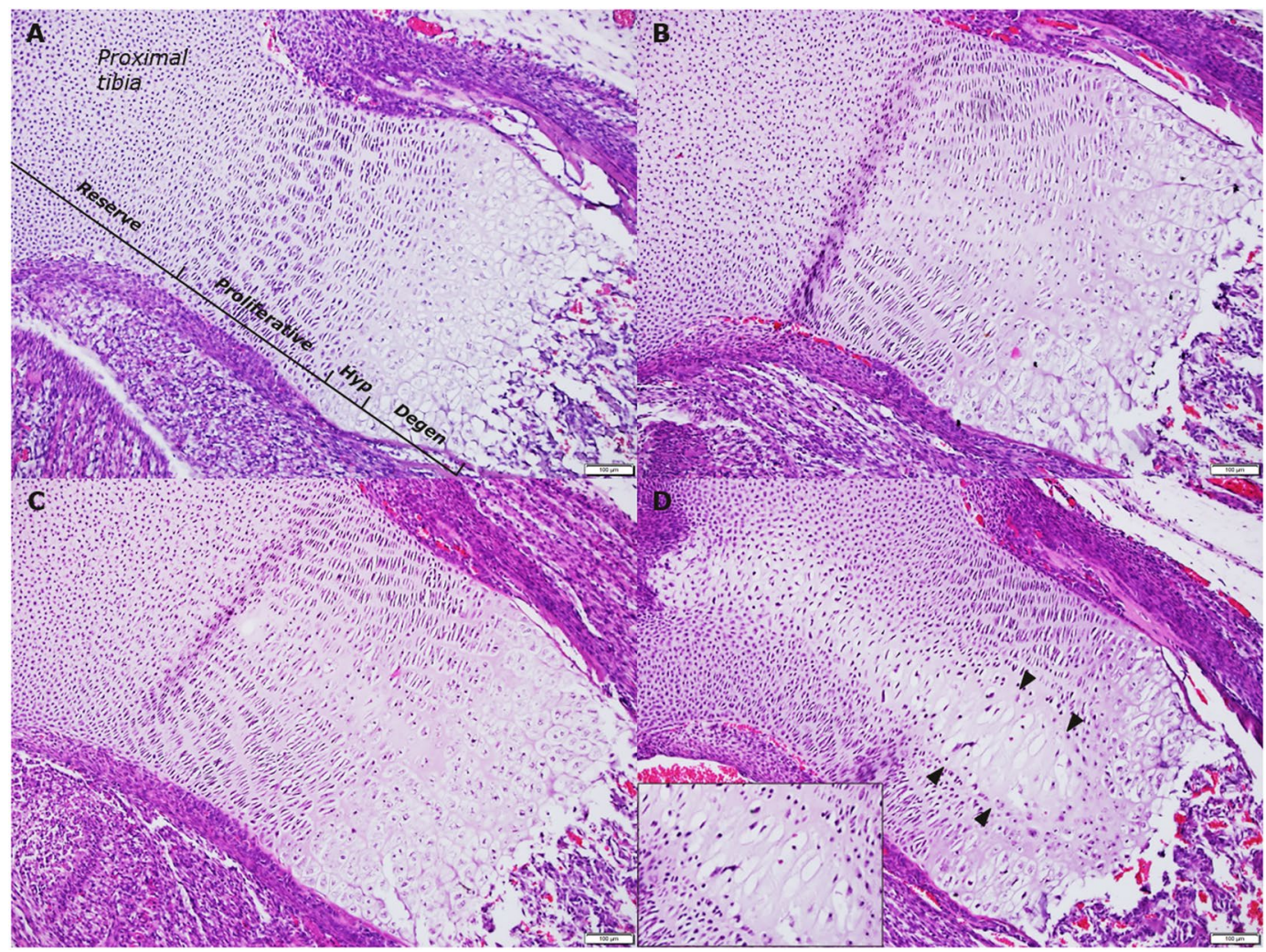

Figure 1. Representative examples of histopathologic changes in foetal tibial growth plates resulting from HIinfusion throughout gestation. (A) Grade 0, no changes. (B) Grade 1, minimal morphological changes centrally, involving all zones except reserve zone. (C) Grade 2, moderate morphological changes centrally, involving all zones except reserve zone. (D) Grade 3, severe morphological changes centrally (arrowheads), involving all zones, with loss of columnar organisation of chondrocytes, the few remaining chondrocytes appearing hypertrophic with pyknotic nuclei (insert). Degen, degenerative; Hyp, hypertrophic. Magnification: x100; insert $\mathrm{x} 200$.

plates and bone cells, and that these changes would be more severe when hypoglycaemia extended throughout gestation compared with only until end of organogenesis.

The investigational strategy builds on our animal model of maternal hypoglycaemia induced by continuous infusion with human insulin (HI) lasting either throughout gestation until termination on GD20 or until GD17 (covering the embryonic period and organogenesis), followed by three days without insulin-infusion $(\text { GD17-20) })^{6}$.

We used four main approaches: A descriptive histologic evaluation, quantitative histology, gene expression analysis, and finally, quantification of plasma hormones. The descriptive histologic evaluation consisted of recording any histopathologic disruption of growth plate organisation, including immunohistochemistry to visualise distribution of collagens, as well as in situ hybridisation to evaluate local expression of anti-hypoxic factors in growth plates. The quantitative assessment included stereologic measurement of growth plate volumes, spongious zone volume, and relative osteoblast: osteoclast volume in ossification centres. Gene expression analysis included quantification of genes related to growth plate structural composition, bone oxygen balance, the IGF-1 pathway, and osteoblast/osteoclast differentiation and activity. Major hormones known to influence bone metabolism such as corticosterone, IGF-1, and thyroid hormones were measured.

The data from this study will enable a better understanding of the pathogenesis of foetal skeletal changes resulting from continuous hypoglycaemia throughout gestation to GD20 (HI-EoGest) versus hypoglycaemia lasting only until end of organogenesis on GD17 (HI-EoOrg) in non-diabetic animals.

\section{Results}

Descriptive histology. Histopathologic evaluation. Morphological changes localised the central longitudinal area of the growth plate were observed in the foetal tibial growth plates of the HI-infused groups (Fig. 1). All foetuses receiving HI-infusion throughout gestation (HI-EoGest), showed changes, which were severe and extended across all growth plate zones (Fig. 1d). The changes were characterised by fewer chondrocytes, which had lost their columnar organisation and displayed pyknotic nuclei and/or hypertrophy. Additionally, borders between the four growth plate zones (resting, proliferative, hypertrophic, degenerative) were irregular and less well-defined; particularly between the hypertrophic and degenerative zones and at the boundary between the degenerative growth plate zone and spongious zone of the primary ossification centre. 

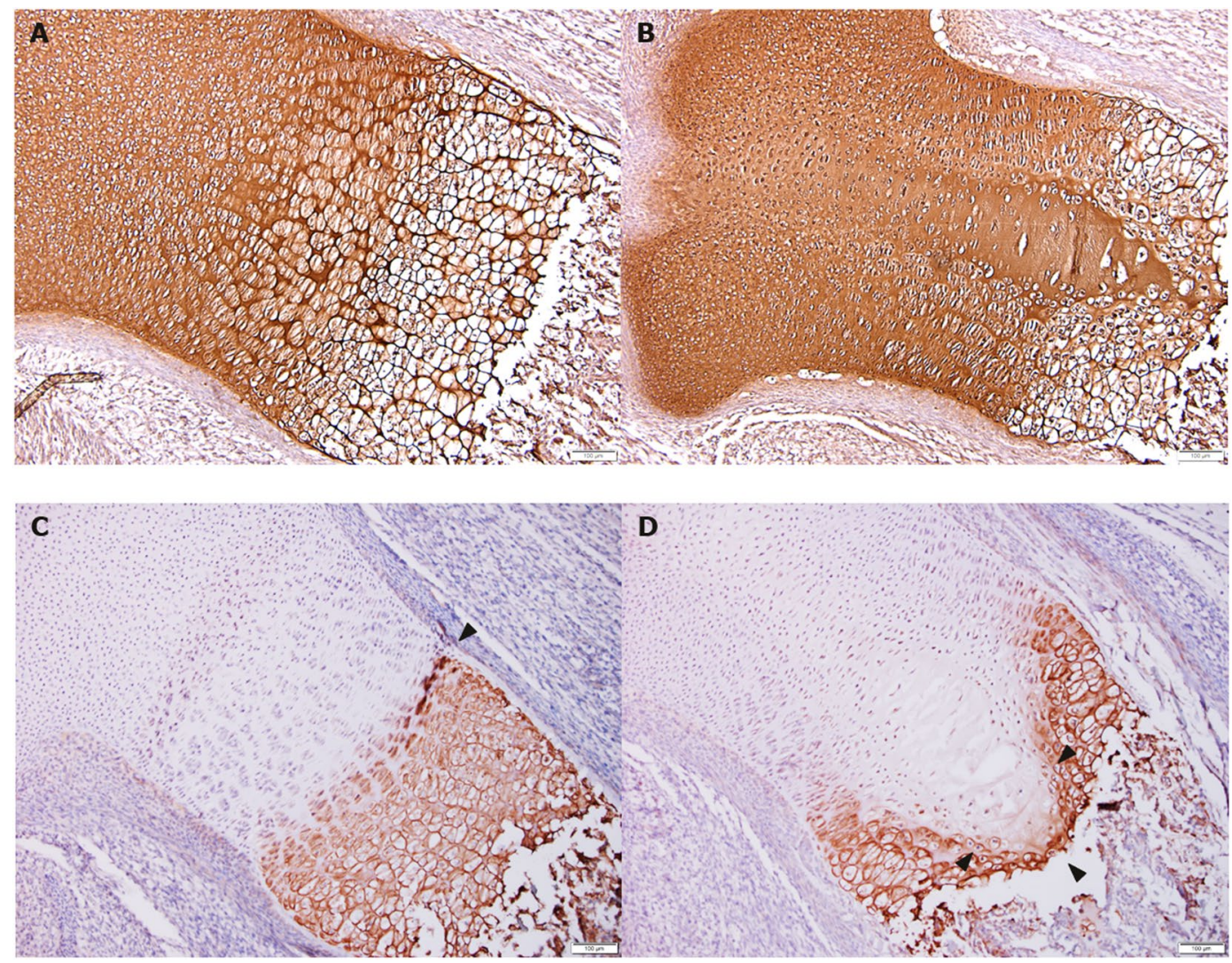

Figure 2. Immunohistochemical staining for collagen II $(\mathbf{A}, \mathbf{B})$ and collagen $X(\mathbf{C}, \mathbf{D})$ in foetal tibial growth plates. Sections stained for collagen II and X (dark brown). (A) CTRL: No histopathologic changes, normal distribution of collagen II throughout the growth plate, (B) HI-EoGest: Grade 3 changes, normal distribution of collagen II including the central area. (C) CTRL: No histopathologic changes. Peri-cellular collagen X staining between hypertrophic zone chondrocytes, clearly defining the boundary between proliferative and hypertrophic zones (arrowhead), (D) HI-EoGest: Grade 3 changes. Peri-cellular collagen X staining distally in the growth plate, no clear distinction of cell types and no central staining, resulting in a "V"-shaped staining pattern (arrowheads) pushing into hypertrophic, degenerative, and spongious zones. (Magnification: x100).

In contrast, when infused only until end of organogenesis (HI-EoOrg) approximately half the foetuses displayed histopathologic changes, and these were of moderate grade (Fig. 1c). Compared to CTRLs, the changes in the HI-EoOrg group were more pronounced.

Immunohistochemistry. In controls, extracellular collagen II staining was observed throughout the growth plates (Fig. 2a); similarly, in the HI-EoGest group, staining was present in the central hypocellular area (Fig. 2b). In controls, collagen $\mathrm{X}$ distribution in growth plates clearly defined the boundary between proliferative and hypertrophic zones through positive staining of peri-cellular regions of hypertrophic chondrocytes (Fig. 2c). While the HI-EoGest showed a similar pattern, the cell types were not easily recognized, and the positive zone was markedly disordered (Fig. 2d).

In situ hybridization. HI-EoGest foetal growth plates had pronounced positive staining for the pro-angiogenic factors HIF- $1 \alpha$ and VEGF-A, locally restricted to the margin of the histopathologic changes (Fig. 3b,d). No positive staining was observed in controls (Fig. 3a,c), except for a few scattered positive cells in the degenerative zone and peri-articular cartilage in some of the control animals, which were not easily recognisable even at $100 \mathrm{x}$ magnification. Additionally, the peri-articular cartilage of foetal tibial and meta-tarsal joints displayed a markedly local increased HIF-1 $\alpha$ expression (Fig. 3e-h). VEGF-A showed a similar expression pattern (data not shown).

As some basal expression of HIF-1 $\alpha$ and VEGF-A is expected in growth plates, the lack of positive staining is likely due to the sensitivity being too low to detect this; however, since the staining was very marked in the cells surrounding the changes, showing a pronounced increase versus controls, this is not considered to significantly affect the results.

Quantitative histology. Stereologic volume quantification. Growth plates. HI-infusion throughout gestation (HI-EoGest) resulted in decreased total growth plate volume $(\mathrm{p}=0.0001$, Fig. $4 \mathrm{a})$ and reduced reserve, proliferative, and degenerative zone volumes ( $\mathrm{p}<0.0001-0.003$, Fig. $4 \mathrm{~b}-\mathrm{e})$. Disproportionately decreased total growth plate and zone volumes were reflected by increased relative volumes of the proliferative and hypertrophic zones $(\mathrm{p}=0.0192 ; \mathrm{p}=0.0239)$, but conversely decreased the relative volume of the degenerative zone $(\mathrm{p}=0.0436$, 

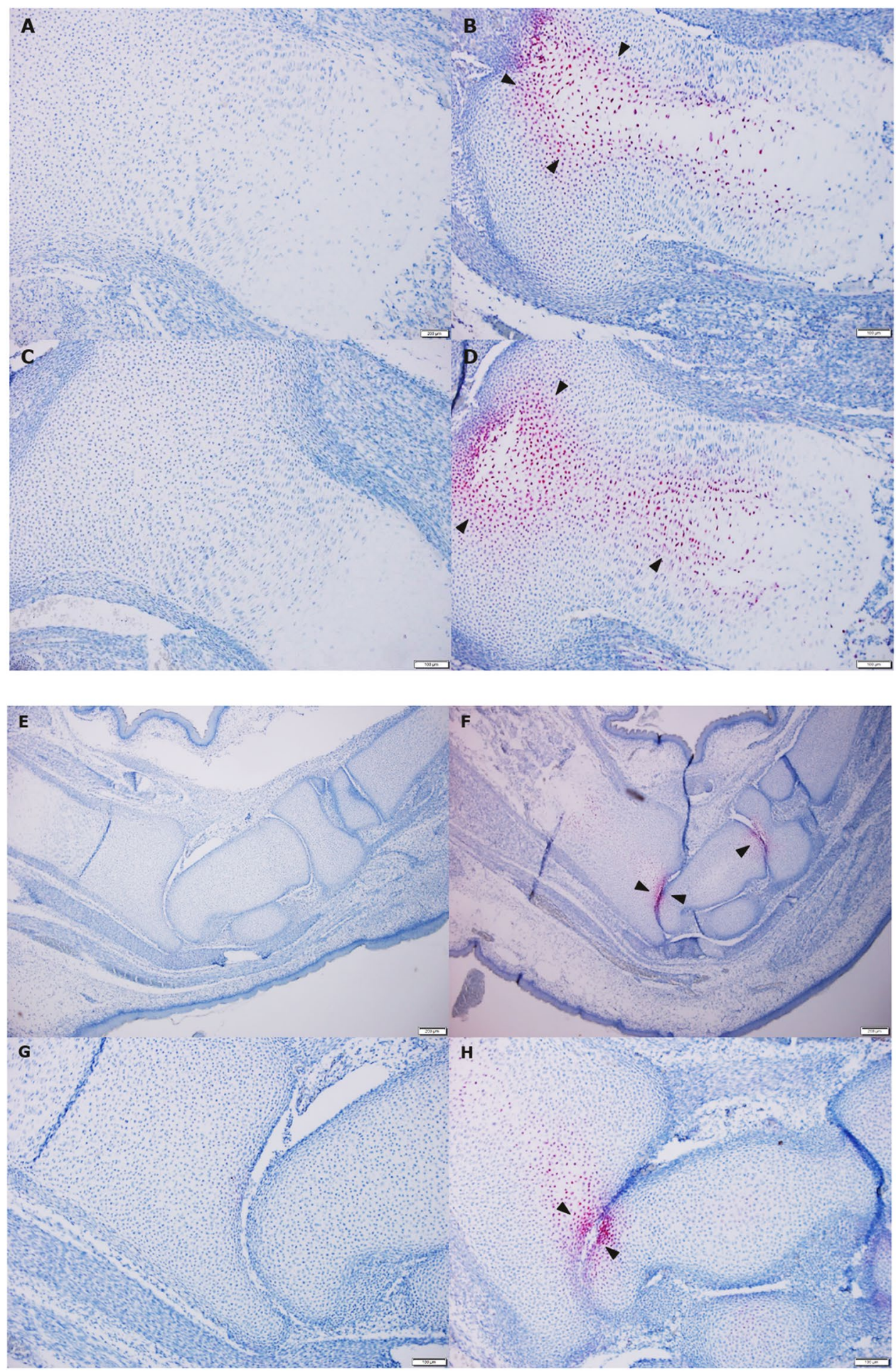

Figure 3. In situ hybridization for VEGF-A and HIF-1 $\alpha$ in foetal tibial growth plates (A-D) and meta-tarsal joints (E-H). Sections stained for VEGF-A and HIF-1 $\alpha$ (pink). Proximal tibial growth plate: (A) CTRL: No histopathologic changes or staining, (B) HI-EoGest: Grade 3 changes and positive intracellular VEGF-A staining (arrowheads) in the cells at the periphery of the changes, (C) CTRL: No histopathologic changes or staining, (D) HI-EoGest: Grade 3 changes and positive intracellular HIF-1 $\alpha$ staining (arrowheads) in the

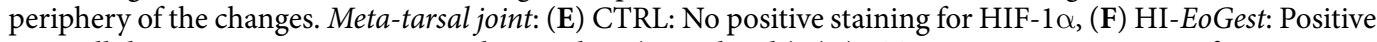
intracellular HIF-1 $\alpha$ staining in articular cartilage (arrowheads), (G) CTRL: No positive staining for HIF- $1 \alpha$, (H) HI-EoGest - Positive intracellular HIF-1 $\alpha$ staining (arrowheads) in articular cartilage. (A-D, G + H, x100 magnification; $\mathrm{E}+\mathrm{F}, \mathrm{x} 40$ magnification). 

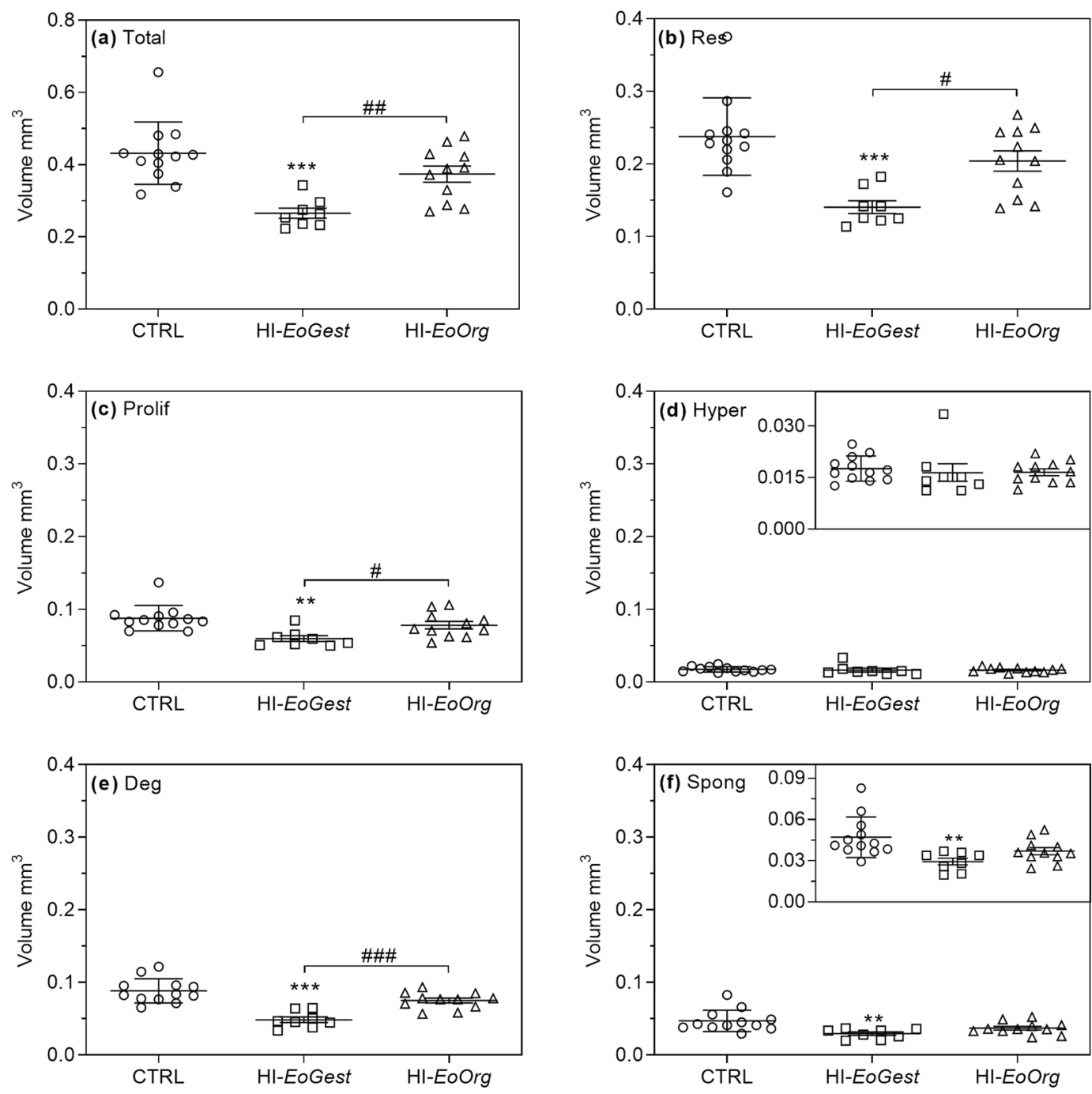

Figure 4. Absolute total growth plate and zone volumes in foetal long bones. Means $\pm S D$ and individual values. Absolute volumes of (a) Total growth plate, (b) (Res)erve zone, (c) (Prolif)erative zone, (d) (Hyper) trophic zone, (e) (Deg)enerative zone and (f) (Spong)ious zone. Inserts (d,f): Enlarged scale. **p $<0.01$, ${ }^{* * *} \mathrm{p}<0.001$ versus CTRL, ${ }^{\#} \mathrm{p}<0.05,{ }^{\# \#} \mathrm{p}<0.01,{ }^{\# \# \#} \mathrm{p}<0.001$ HI-EoOrg versus HI-EoGest. One-way ANOVA with post hoc Tukey's multiple comparisons test.

Fig. 5). Recovering normoglycaemia after organogenesis (HI-EoOrg) resulted in increased total growth plate volume compared to the HI-EoGest $(\mathrm{p}=0.0140)$, reaching control levels; similarly, the reserve, proliferative, and degenerative zone volumes did not differ from controls $(\mathrm{p}=0.0007-0.0490)$. Relative zone volumes were in-between group HI-EoGest and CTRL levels.

Spongious zone. The absolute spongious zone volume was decreased in HI-EoGest animals $(\mathrm{p}=0.0042)$, whereas volumes for the HI-EoOrg group ranged between HI-EoGest and CTRL groups (Fig. 4f). The ratio of spongious zone: total growth plate volumes was not affected by HI-infusion (mean ratios $0.10-0.11 \pm 0.01-0.02$ for all three groups; mean $\mathrm{CE} \leq 0.05$ and $\mathrm{SD} \leq 0.01$ for all).

Osteoblasts and osteoclasts. Immunohistochemical staining of osteoblasts nuclei/osteoclasts cytoplasm is illustrated in Supplementary Fig. S1. The ratio of osteoblast: osteoclast volumes in primary ossification centres was not affected by HI-infusion, regardless of duration (mean ratios CTRL $1.74 \pm 0.32 ; \mathrm{n}=13$, EoGest $1.71 \pm 0.41$, $\mathrm{n}=7$, HI-EoOrg $1.73 \pm 0.34, \mathrm{n}=11$; mean $\mathrm{CE} \leq 0.02 \pm 0.1$ for all groups).

Gene expression analysis. $\quad R T-q P C R$ quantification of foetal bone tissue mRNA levels. HI-infusion did not alter mRNA levels of cartilage collagens, hypoxia-related genes, the structurally related gene comp, genes related to the IGF-1 pathway nor osteoblast/osteoclast differentiation and activity (data not shown). In contrast, mRNA level of Matn-3, an additional structurally related gene, was decreased in HI-EoGest animals ( $\mathrm{p}=0.0222$, data no shown), whereas recovering from hypoglycaemia after organogenesis (HI-EoOrg) resulted in normal mRNA level of this gene. 


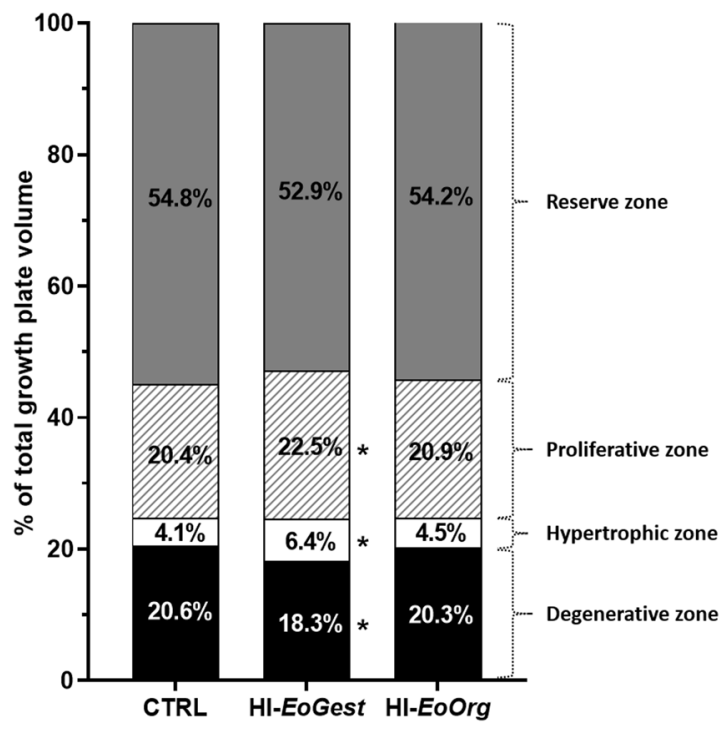

Figure 5. Relative volumes of tibial growth plate zones shown as percentage of total growth plate volume. Absolute zone volumes related to total growth plate volumes (group means). Each column represents $100 \%$ of the growth plates volumes. CTRL, $\mathrm{n}=12$; HI-EoGest, $\mathrm{n}=8$; HI-EoOrg, $\mathrm{n}=11$. ${ }^{*} \mathrm{p}<0.05$ versus CTRLs. Oneway ANOVA with post hoc Tukey's multiple comparisons test.
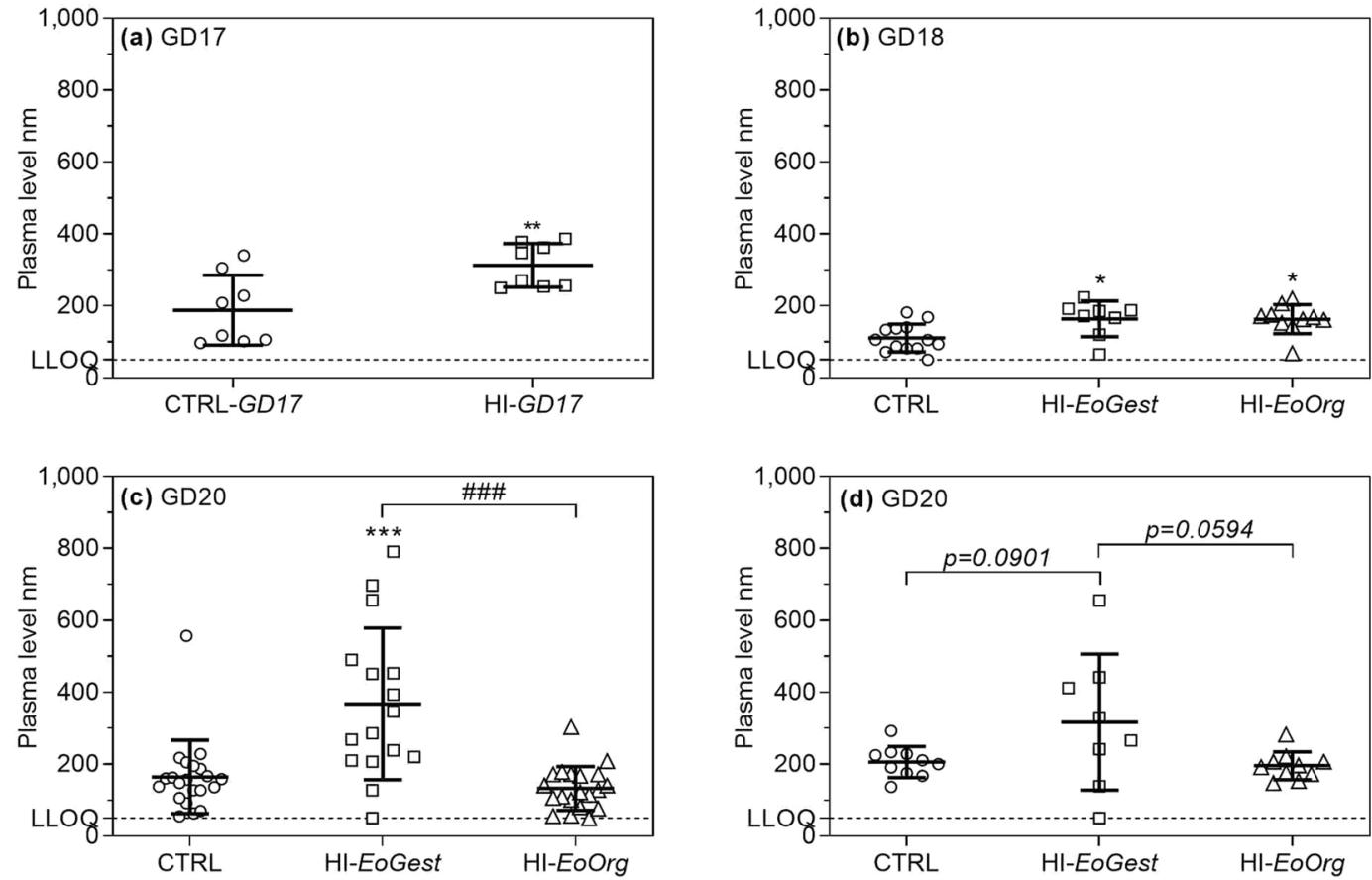

Figure 6. Plasma corticosterone levels: maternal (a-c) and foetal (d). Means $\pm S D$ and individual values. (a) Maternal levels at sacrifice on GD17. (b) Maternal levels on GD18. (c) Maternal levels at sacrifice on GD20. (d) Foetal litter levels on GD20. Dotted line: LLOQ (lower limit of quantification: $50 \mathrm{nmol} / \mathrm{l}$ ). Four samples assayed $<$ LLOQ and were set to $50 \mathrm{nmol} / \mathrm{L}$ (dams: 1 CTRL, GD18; $1 \mathrm{HI}-$ EoGest and $1 \mathrm{HI}-E o O r g$, GD20. Litter: $1 \mathrm{HI}-$ EoGest, GD20). $* \mathrm{p}<0.05, * * \mathrm{p}<0.01, * * * \mathrm{p}<0.001$ versus CTRLs. ${ }^{\# \#} \mathrm{p}<0.001$ HI-EoOrg versus HI-EoGest. (a) Unpaired two-tailed t-test, (b-d) One-way ANOVA with post hoc Tukey's test.

Plasma hormone levels. Maternal and foetal corticosterone. Maternal corticosterone levels were increased in HI-infused animals on GD17 (HI-GD17; p < 0.0082) and GD18 (HI-EoGest, $\mathrm{p}=0.0241 ; \mathrm{HI}-\mathrm{EoOrg} \mathrm{p}=0.0172$. Figure $6 \mathrm{a}, \mathrm{b})$, with no difference between these groups. On GD20, HI-EoGest corticosterone levels remained elevated, whereas HI-EoOrg levels had returned to normal ( $\mathrm{p}<0.0001$, Fig. 6c). 
Litter corticosterone levels on GD20 differed between the three groups overall $(\mathrm{p}=0.0478)$ but were not significantly different between individual groups (Fig. 6d). Exclusion of one HI-EoGest outlier (<LLOQ) strengthened the association $(\mathrm{p}=0.0030)$ such that HI-EoGest levels were higher on GD20 compared to CTRLs and HI-EoOrg $(\mathrm{p}<0.01)$.

As maternal and litter corticosterone levels in the HI-EoGest group were elevated on GD20, we tested the correlation between levels in individual dams and corresponding litter and this was strong (Pearson $\mathrm{r}=0.834$, $\mathrm{p}=0.010$, Supplementary Fig. S2).

Maternal and foetal IGF-1. Maternal IGF-1 levels were lower on GD17 (HI-GD17 p =0.0276, Supplementary Fig. S3). In contrast, on GD18 (24h after infusion-stop in group HI-EoOrg), IGF-1 levels did not differ between groups though there was a trend for decreased levels in HI-EoGest (Supplementary Fig. S3).

On GD20, HI-EoGest IGF-1 levels were reduced ( $\mathrm{p}=0.0003$, Supplementary Fig. S3), whereas after an infusion-free period from GD17-GD20 levels were normal (HI-EoOrg, $\mathrm{p}=0.5417$ ).

Foetal IGF-I levels were unaffected in HI-infused animals on GD20 (Supplementary Fig. S3).

Maternal thyroid hormones and TSH. Maternal TSH levels were unaffected by insulin-infusion (data not shown); likewise, for $\mathrm{T}_{3}$, although there was a trend towards increased levels in HI-GD17 on GD17 (Supplementary Fig. S4). $\mathrm{T}_{4}$ levels were increased by HI-infusion (0.0009, Supplementary Fig. S4), a similar picture was seen $24 \mathrm{~h}$ later (GD18) (HI-EoGest, $\mathrm{p}=0.0112$; HI-EoOrg, $\mathrm{p}=0.0064$ versus CTRL. Suppl. Fig. 4). On GD20, HI-EoGest group $\mathrm{T}_{4}$ levels remained elevated $(\mathrm{p}<0.0001)$, whereas HI-EoOrg group levels had returned to control levels (Supplementary Fig. S4).

\section{Discussion}

We have previously used $\mathrm{HI}$-infusion in a pregnant non-diabetic rat model to investigate the effects from continuous hypoglycaemia on foetal skeletal development. We showed that maternal hypoglycaemia throughout gestation delays foetal growth and skeletal development, while hypoglycaemia only until after completion of organogenesis (GD17) allows for near-normal development ${ }^{6}$. The mechanisms underlying these developmental changes are not fully understood. In order to elucidate this, we extended our investigation to assess for histopathologic changes and potential regulatory players in foetal bone development, based on the hypothesis that hypoglycaemia disrupts regulation of the skeletal micro-environment. The present study clearly shows that continuous hypoglycaemia throughout gestation causes pronounced histopathologic changes in tibial growth plates including disproportionately altered zone volumes. These changes are highly similar to changes caused by hypoxia, leading us to propose hypoglycaemia-induced hypoxia as a pivotal mechanism (Supplementary Fig. S5). When hypoglycaemia lasted only until GD17, histopathologic changes were less pronounced and growth plate volumes normal.

The observed severely disrupted ECM architecture in central foetal growth plates following hypoglycaemia throughout gestation may have resulted, at least in part, from decreased expression of the ECM protein MATN-3, essential to structural integrity of the chondrocyte scaffold ${ }^{7}$. In contrast, expression of COMP, collagen IX, and collagen II were unaffected, although similarly important for growth plate structural integrity through the formation of interconnected complexes with MATN- $3^{7,8}$. In murine growth plates, ablation of any one of these proteins causes a longitudinal, central disruption of the columnar chondrocyte organisation and decreased cell numbers, occasionally accompanied by hypertrophic chondrocytes with pyknotic nuclei ${ }^{9-13}$. These changes are very similar to the appearance and confinement of the histopathologic findings in the current study and those in studies by others following growth plate hypoxia ${ }^{14-17}$, hence the rational for our hypothesis. Reversely, MATN-3 decreases may be secondary to a hypoxia-induced disruption of the ECM architecture. The less pronounced chondrocyte disorganisation and normal MATN-3 expression after hypoglycaemia only until GD17 indicates that changes have either partly recovered after GD17 or the development has been attenuated. From this study, it cannot be concluded which of these scenarios take place, as growth plates were not evaluated on GD17.

In support of our interpretation, extended hypoglycaemia increased expression of the hypoxia-protecting factors, HIF-1 $\alpha$ and VEGF-A, at the boundaries immediately surrounding the central histopathologic changes. Strikingly similar histopathologic changes were recorded in growth plates following ablation of either HIF-1 $\alpha$ or VEGF-A in mice ${ }^{14-17}$ and vice versa, exposure of cultured chondrocytes to hypoxia increases expression of these factors ${ }^{18-20}$. Moreover, in mice, growth plate hypoxia induced by chondrocyte-specific inactivation of the HIF-1 $\alpha$ gene increases VEGF-A expression at the periphery of the accompanying central growth plate changes ${ }^{17}$, similar to our findings. In line with this, histological growth plate changes were confined to the longitudinal central area, which is particularly vulnerable to hypoxia, being avascular and relying on passive oxygen diffusion from adjacent blood vessels ${ }^{17,21}$. This suggests that the histologic growth plate changes seen here are due to central hypoxia, which in turn elicits a local counter-regulatory increase of HIF-1 $\alpha$ and VEGF-A levels.

On GD20, the increased maternal corticosterone levels following hypoglycaemia throughout gestation are likely caused by the hypoglycaemia, and is a known counter-regulatory response, increasing glucose-output from the liver ${ }^{22}$, also explaining changes in maternal IGF-1 and thyroid levels ${ }^{23-25}$. Since maternal and litter plasma corticosterone levels correlated in the hypoglycaemic animals, we speculate that increased maternal corticosterone level in turn exposed the foetus to increased levels through trans-placental transport ${ }^{26}$. Subsequently, foetal corticosterone may have impeded vascularisation in the growth plate adjacent to the metaphyseal area. Corticosteroid exposure has been shown to decrease production of VEGF-A by growth plate chondrocytes in vitro and in vivo ${ }^{27,28}$, and induce growth plate hypoxia by decreasing density of adjacent blood vessels in mice and piglets $^{27,29}$. This is also in line with our observation of increased HIF-1 $\alpha$ and VEGF-A mRNA levels in foetal tibial and meta-tarsal joint articular cartilage, which is equally avascular and vulnerable to decreased peri-articular angiogenesis following increased blood corticosteroid levels in mice ${ }^{27,30,31}$. The observed secondary increases in anti-hypoxia factors may be a compensatory response to corticosterone-induced hypoxia. In line with this, 
ending hypoglycaemia on GD17 and normalising corticosterone levels may have interrupted the progression of the histopathologic changes.

In the above studies, no central histopathologic changes were observed in growth plates following corticosteroid exposure, however, this does not invalidate the present working hypothesis. Unlike the present study, administration was postnatal, and it is probable that the developing skeleton may be more vulnerable to corticosteroid exposure; counter-regulatory measures may also differ. Furthermore, temporary dosing was used, whereas in the present study, foetuses were exposed to high corticosterone levels, presumably, from conception.

Hypoglycaemia throughout gestation decreased total growth plate volume as anticipated based on previously observed shortened tibia length in litter-mates (foetuses from the same litters used for other investigations) ${ }^{6}$, which are known effects of glucocorticoid-dosing in rats and rabbits ${ }^{32-34}$. The disproportionate zone volume changes may be linked to reduced local VEGF-A levels. VEGF-A inactivation in growing mice expands the hypertrophic zone in tibial growth plates ${ }^{35}$, and chondrocyte-specific VEGF-A knock-out increases hypertrophic and decreases degenerative zone lengths in mouse embryo tibial growth plates (through delayed maturation/removal of hypertrophic chondrocytes $)^{15}$. This is in line with the hypothesis of a key role of hypoxia in the present changes. Decreased MATN-3 expression may also be implicated, since MATN-3 KO promotes chondrocyte hypertrophy in mouse embryos ${ }^{10,36}$; this is reflected by an increased hypertrophic zone length in tibial growth plates following premature maturation of proliferative into hypertrophic chondrocytes ${ }^{10}$. In contrast to hypoglycaemia lasting throughout gestation, hypoglycaemia ending on GD17 resulted in only slightly decreased total and separate growth plate volumes, which were not statistically different from controls; this was surprising given the shorter tibial length in litter-mates ${ }^{6}$. However, since this decrease in tibial length was less pronounced than after hypoglycaemia throughout gestation ${ }^{6}$, it possibly reflects delayed bone growth prior to GD17 extending beyond GD17, allowing only for an attenuation of the decrease.

In addition to a potential primary role of hypoxia in the development of the histopathologic growth plate changes, hypoglycaemia may also play an important role. The growth plate is a highly metabolic tissue, relying on anaerobic glycolysis ${ }^{37}$, and glucose serves as an essential source of energy ${ }^{38}$. Consequently, central chondrocytes may die due to glucose insufficiency and impaired glycolysis decreasing the energy supply. Apoptosis was seen despite local up-regulation of HIF-1 $\alpha$, which is known to increase expression of glucose transporters and enzymes of the glycolytic pathway ${ }^{39-41}$. However, this does not exclude a concurrent role of hypoxia in the changes; changes may be caused by a combination of hypoglycaemia and hypoxia.

Our data suggests that during foetal development, the growth plates are particularly vulnerable to maternal hypoglycaemia, more so than the ossification process, regardless of duration. High corticosteroid levels are known to decrease osteoblast and increase osteoclast activity and number/life-span in bone, leading to decreased bone formation and increased bone resorption ultimately reducing bone mineral density ${ }^{30,42-44}$. However, following hypoglycaemia throughout gestation, parameters relating to the bone mineralisation process did overall not change, in line with our previous findings of unaffected tibial peak mineral density in litter-mates in this group ${ }^{6}$.

Our investigation has some limitations; firstly, animal models are not necessarily translatable to humans, which should be kept in mind when interpreting the results. Moreover, pregnant diabetic women would not be continuously hypoglycaemic following insulin-dosing. Secondly, the rats are not diabetic, therefore the influence of counter-regulatory responses might differ in diabetics. This model was not intended as an animal model of diabetes, but rather, a model of experimentally insulin-induced hypoglycaemia, to be used as a means of understanding mechanisms involved in normal skeletal development.

This strengthens the study in several aspects including that it provides a stable controlled model in which all animals were similar at the outset and unaffected by disease. It also allows for investigation of changes to foetal skeletal development during hypoglycaemic conditions. Moreover, to better understand duration of continuous hypoglycaemia on foetal skeletal development we included both a group with insulin-induced hypoglycaemia throughout gestation as well as a group with hypoglycaemia only until completion of organogenesis (GD17) in order to also study prevention of foetal changes. Additionally, insulin-infusion was well tolerated and less stressful than the otherwise necessary multiple daily dosing needed to approach continuous hypoglycaemia, allowing for better animal welfare. Finally, the breadth of analytical methods employed provides linkage between observed histopathological changes and potential regulatory factors.

In summary, this experimental rat model of insulin-induced hypoglycaemia on foetal skeletal development shows pronounced effects on growth plate organisation; however, discontinuation of insulin-infusion after organogenesis allows for a partly normal development. We speculate that the skeletal changes are induced by hypoxia secondary to increased corticosterone levels (Supplementary Fig. S5). The hypoglycaemia induces a counter-regulatory increase in maternal plasma corticosterone levels, which in turn increases foetal corticosterone levels, decreasing oxygen supply to central growth plates, causing hypoxia, reflected by local counter-regulatory increases in anti-hypoxic factors and hypoxia-like histopathologic changes. Future follow-up investigations to validate this hypothesis could include measurement of tissue oxygen level in the growth plates, as well as histologic evaluation of: i) metaphyseal vascularisation (e.g. by immunostaining for endothelial cell markers), ii) local HIF-1 $\alpha$ and VEGF-A protein levels (e.g. by immunohistochemistry), to determine if they mirror the mRNA changes, and iii) the apoptotic phenotype of the growth plate chondrocytes (e.g. by TUNEL assay).

In conclusion, our study underlines the importance of sufficient foetal glucose availability during late gestation for support of normal skeletal development.

\section{Materials and methods}

Experimental design. The study design is presented in Fig. 7 and described in detail elsewhere ${ }^{6}$, but briefly, female Sprague-Dawley rats (approximately ten weeks old) were continuously infused with either HI or vehicle, starting one week prior to mating (Day 1). 


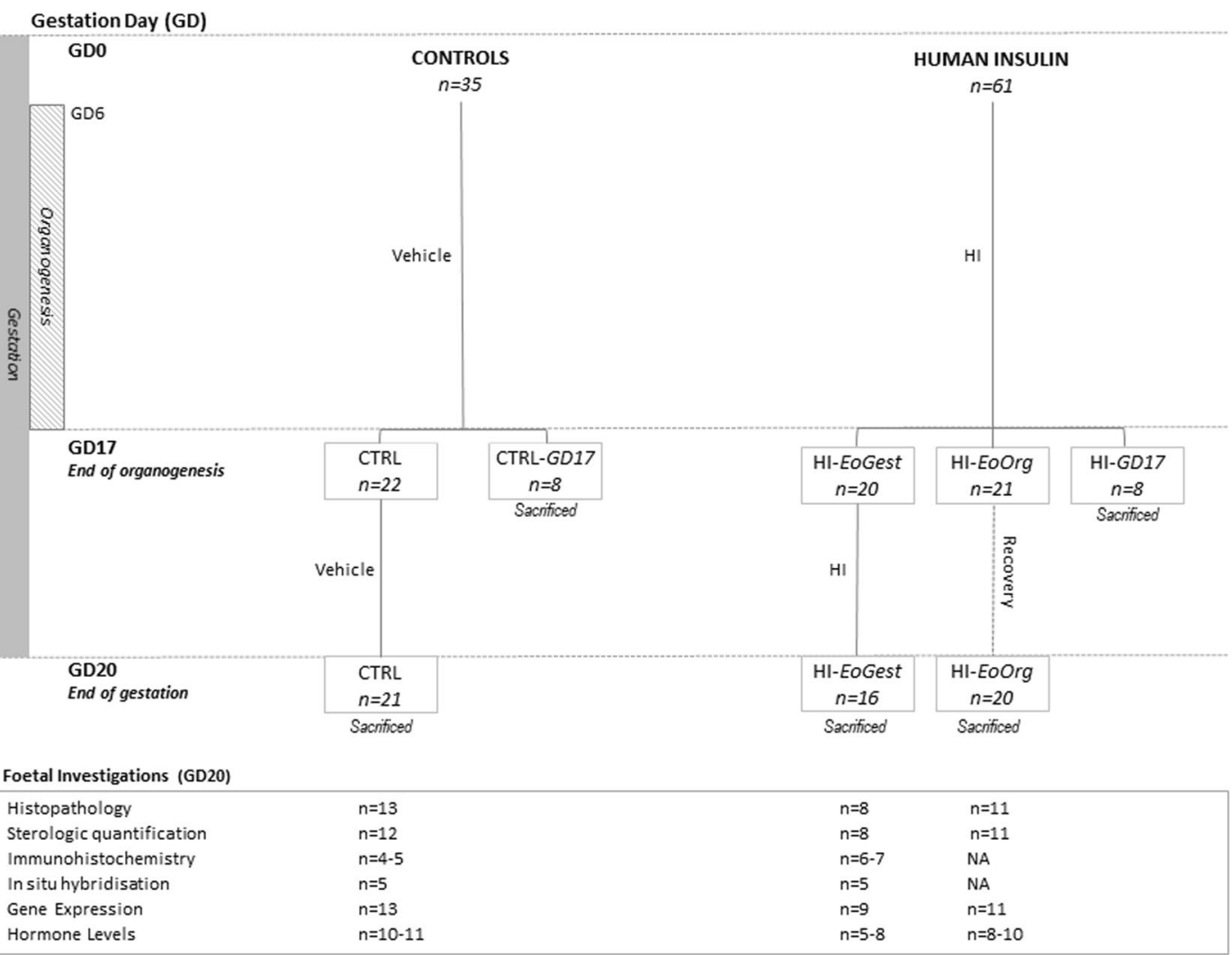

Figure 7. Study design including time-frames, investigations and animal numbers. Experimental groups received infusion with human insulin (HI) until end of gestation (EoGest) i.e. gestation day (GD) 20 (HI-EoGest group) or end of organogenesis (EoOrg) on GD17 (HI-EoOrg group). NA, not analysed.

The two experimental groups were: HI-infusion throughout and until end of gestation i.e. until gestation day (GD)20 (HI-EoGest); HI-infusion only until GD17 (i.e. approximate end of organogenesis) followed by a three-day infusion free period (HI-EoOrg). Control animals were infused with vehicle until GD20. Animals which died or were terminated prematurely were excluded; a total of 44 animals were infused with HI (16 received HI-infusion until GD20 and 20 until GD17); 29 were infused with vehicle. On GD17 eight animals from the HI-infused and control group were sacrificed for maternal blood sampling, these sub-groups were termed HI-GD17 and CTRL-GD17, respectively. At the end of the study (GD20) all remaining animals were sacrificed, and various parameters assessed (described below).

It has been confirmed that insulin-infusion decreased maternal blood glucose levels as intended, from $3 \mathrm{~h}$ after infusion-start ${ }^{6}$; at the end of study/sacrifice HI-EoGest foetal blood glucose levels were also decreased.

All procedures involving live rats have been ethically approved by the Animals (Scientific Procedures) Act 1986 (ASPA) and United Kingdom Secretary of State and performed according to the ARRIVE guidelines, Directive 2010/63/EU, EC Commission Directive 2004/10, OECD Principles and Good Laboratory Practice, and The Good Laboratory Practice (Codification Amendments Etc.) Regulations 20045-48, as well as Envigo and Novo Nordisk A/S company policies on the care and use of laboratory animals.

Sampling of foetal tissue. Within one hour of sacrifice, one foetus per litter was taken from a mid-horn position (alternating between left/right uterine horn) from approximately half the dams in each group (Fig. 7). From each foetus, the left hind limb was fixed in $10 \%$ neutral buffered formalin and paraffin embedded for histology. The paraffin block was sectioned into $5 \mu \mathrm{m}$ thick lateral longitudinal sections, mounted on glass slides, and stored at $-20^{\circ} \mathrm{C}$ until staining. The starting point was set at the first section where tissue appeared. From and including the first section, every fifth section was stained with haematoxylin and eosin (H\&E) for histopathologic evaluation and quantification of growth plate zone volumes. The next slide in line after each of these sections was used for quantification of osteoblast-osteoclast ratios in the primary ossification centre. Sections were scanned (Aperio AT2 scanner, Leica Biosystems, Nussloch, Germany) and histopathologic evaluations and volume quantifications were performed blinded. From a subset of animals in the CTRL and HI-EoGest group (see below), the next three adjacent slides (i.e. third, fourth, fifth) were used for either immunohistochemistry or in situ hybridization (see below).

Descriptive histology. Histopathologic evaluation. Foetal skeletal histopathologic changes were evaluated using a four-step grading scale based on severity and extent of changes (x10 and x100 magnification). Features for each grade were: Grade 0, no changes; Grade 1, minimal changes and only a slightly increased amount of 
extracellular matrix (ECM) across all zones, except the reserve zone; Grade 2, moderate changes, increased ECM and mild hypertrophy of chondrocytes across all zones, except the reserve zone; Grade 3, severe changes, characterised by increased though remarkably hypocellular ECM. The affected area extending through all growth plate zones.

Immunohistochemistry. To assess structural integrity and demarcation of the hypertrophic zone in tibial growth plates, distribution of collagen II and X was qualitatively evaluated by light microscopy from a subset of animals displaying severe (HI-EoGest group, $\mathrm{n}=6-7$ animals) or no changes (CTRL group, $\mathrm{n}=4-5$ animals). Representative sections, 2-4 sections per animal (slide 3, 4 or 5) were selected for evaluation. Immunohistochemistry was not performed in HI-EoOrg animals.

To identify osteoblasts and osteoclasts in primary ossification centres, tibial sections were stained for osterix (in osteoblast nuclei) and CD68 (localised in lysosome membranes of osteoclasts). Protocols are included in Supplementary Material.

In situ hybridization. To evaluate hypoxia-related changes in foetal growth plates, distribution of anti-hypoxic factors HIF-1 $\alpha$ and VEGF-A was assessed in 2-4 representative sections from animals with severe or no changes (i.e. HI-EoGest and CTRL groups; 5 animals/group). In situ hybridization was not performed in HI-EoOrg animals. mRNA signal was detected using Automated Assay for Ventana Systems. The assay RNAscope 2.5 VS Reagent Kit-RED (322250,) Probe-Rn-Hifla (432289) and Probe-Rn-Vegfa (315369) from Advanced Cell Diagnostics, Inc., Newark, CA, USA.

Quantitative histology. Stereologic quantification of volumes. The sampling method described above resulted in 11-22 H\&E stained sections per foetus that contained growth-plate. Volumes of each growth plate zone and adjacent primary spongious zone were quantified based on the Cavalieri principle ${ }^{49}$. Demarcation of individual zones was defined according to the morphologic criteria of chondrocytes in each zone described by Burdan et al. ${ }^{50}$. NewCAST software (Visiopharm, Hoersholm, Denmark) was used; point-counting was based on preliminary counting on randomized sections, with a minimum 150-200 hit-points in total and a coefficient of error $(\mathrm{CE})<10 \%$. Grid systems used $7 \times 8$ (reserve zone) and $12 \times 12$ points (remaining four zones) per view field (on-screen magnification $\mathrm{x} 100$ ). The sampling fraction was $100 \%$ of growth plate area on each section (shape factor 4, smooth organ). For each zone, volume and mean CE were calculated according to stereologic principles $^{49,51}$.

The primary ossification centre was included in 6-18 sections/foetus. Osteoblast nuclei and osteoclast cytoplasm total volumes were estimated using a grid system with $2 \times 2$ and $5 \times 5$ points per view field (on-screen magnification $\mathrm{x} 400$ ). Sampling fraction was $100 \%$ of the primary ossification centre per section (shape factor 6 , smooth organ). Volumes and mean CE were calculated as above followed by calculation of volume ratio.

Gene expression analysis. $\quad R T-q P C R$ quantification of foetal bone tissue mRNA levels. One foetus per litter was taken from a mid-horn position from the uterine horn (opposite to the above), hind limbs were sectioned at the hip-joint, soft tissue removed by microdissection, bones placed in aluminium foil envelopes, snap frozen in liquid nitrogen, and stored at $-20^{\circ} \mathrm{C}$ until RNA isolation. Total RNA was extracted using Trizol (15596026, Thermo Fisher Scientific, Waltham, MA, USA), purified using RNeasy Mini Kit (74104, QIAGEN AB, Sollentuna, Sweden), and reverse transcribed to cDNA using High-Capacity cDNA reverse Transcription Kit (4374966, Thermo Fisher Scientific). mRNA levels were quantified by Real-Time PCR using QuantStudio 7 Flex Real-Time PCR system and Taqman Gene Expression Assays (Applied Biosystems, Foster City, CA, USA).

Genes investigated included: Col10a1, Col9a1, Col2a1, Comp, Matn-3 (for structural composition of cartilage/ growth plate); Hif-1 $\alpha$, Vegf-A (for regulation of oxygen balance); Trap, Alp, Runx2, Rankl (for osteoblast/osteoclast differentiation and activity); Igf-1, Igf-R, Igfbp-3, Igfbp-5 (IGF-1 pathway). Housekeeping genes: Gapdh and $\beta$-actin.

Triplicate measurements were performed. If the standard deviation (SD) of mean $\mathrm{C}_{\mathrm{T}}$ values exceeded 0.167 and one measurement was a clear outlier, this was removed. If there was no clear outlier, $\mathrm{C}_{\mathrm{T}}$ values from all three measurements were included in the mean. The " $\Delta \Delta \mathrm{C}_{\mathrm{T}}$ Method" was used to calculate relative expression of each target gene. First the target gene $\mathrm{C}_{\mathrm{T}}$ value $\left(\mathrm{C}_{\mathrm{T}}\right.$, target) was normalized to the mean $\mathrm{C}_{\mathrm{T}}$ value of the two housekeeping (reference) genes, $\left(C_{T}\right.$, ref), for each animal and target gene separately $\left(\Delta C_{T}=C_{T}\right.$, target- $C_{T}$, ref). Thereafter, mean $\Delta \mathrm{C}_{\mathrm{T}}$ and SD were calculated for each target gene within each group. The $\Delta \Delta \mathrm{C}_{\mathrm{T}}$ value was then calculated by normalizing the group mean $\Delta \mathrm{C}_{\mathrm{T}}$ for each test group to the mean $\Delta \mathrm{C}_{\mathrm{T}}$ of the control group $\left(\Delta \Delta \mathrm{C}_{\mathrm{T}}=\right.$ mean $\Delta \mathrm{C}_{\mathrm{T}}$, test group - mean $\Delta \mathrm{C}_{\mathrm{T}}$, control group). Data are reported as $-\Delta \Delta \mathrm{C}_{\mathrm{T}}$ values.

Quantification of hormones in plasma. Sampling of blood for quantification of plasma hormone levels was performed on the following days:

Maternal: on GD17, groups CTRL-GD17 $(\mathrm{n}=8)$ and HI-GD17 $(\mathrm{n}=8)$ were sacrificed and sampled. On GD18, CTRL, HI-EoGest, and HI-EoOrg groups were sampled (approximately $24 \mathrm{~h}$ after infusion-stop in the HI-EoOrg animals). On GD20, at sacrifice, all dams and litters (pooled blood) were sampled.

Plasma corticosterone. Plasma corticosterone (maternal and foetal) was quantified using a commercial ELISA kit (EIA-5186, DRG International, Springfield, New Jersey, USA). Plasma was stored at $-20^{\circ} \mathrm{C}$ before analysis. Lower limit of quantification (LLOQ): $50 \mathrm{nM}$. 
Plasma insulin-like growth factor 1 (IGF-1). Plasma was pre-treated with a proprietary "releasing agent" to displace all IGF-1 bound to binding protein. IGF-1 (maternal and foetal) was quantified using a commercially available ELISA kit (E25, Mediagnost, Reutligen, Germany). LLOQ: $0.029 \mathrm{ng} / \mathrm{ml}$.

Plasma thyroid hormones and thyroid stimulating hormone (TSH). Plasma thyroid hormone $\left(\mathrm{T}_{4}\right.$ and $\mathrm{T}_{3}-$ maternal) and TSH (maternal and foetal) was quantified using commercially available assays (STTHMAG-21K; Milliplex MAP Rat TSH Pituitary Panel (RPTMAG-86K, both Millipore (U.K) Ltd, Hertfordshire, UK). LLOQ: T4 $(1.95 \mathrm{ng} / \mathrm{ml})$, T3 $(0.391 \mathrm{ng} / \mathrm{ml})$ and TSH $(123 \mathrm{pg} / \mathrm{ml})$. Measurements were performed in duplicate. Reported means are from individual dams/litters.

Statistics. Histopathology. Incidence and severity of histopathologic changes were compared between groups using Fishers exact test.

Stereology. In each animal total growth plate volume was calculated as the sum of each zone (reserve, proliferative, hypertrophic, degenerative). Also calculated for each zone were: the volume of each zone relative to total growth plate volume; the ratios of spongious zone: growth plate and osteoblast: osteoclast volume. Group means were compared using one-way ANOVA.

qPCR. For each target gene, $\Delta \Delta \mathrm{C}_{\mathrm{T}}$ values were compared using one-way ANOVA.

Plasma hormone levels. Comparisons between groups were analysed using one-way ANOVA unless more than one sample/group was $<$ LLOQ, in which case Kruskal-Wallis with a post hoc Dunn's multiple comparisons test was used. Results from GD17 were compared using an unpaired two-tailed t-test.

Overall, when one-way ANOVA was used and when there was a significant effect of group, a post hoc Tukey's multiple comparisons test was performed. A p-value $<0.05$ was considered statistically significant.

\section{Data availability}

Data are available upon request.

Received: 12 November 2019; Accepted: 16 March 2020;

Published online: 27 March 2020

\section{References}

1. Jensen, V. F. H., Molck, A. M., Lykkesfeldt, J. \& Bogh, I. B. Effect of maternal hypoglycaemia during gestation on materno-foetal nutrient transfer and embryo-foetal development: Evidence from experimental studies focused primarily on the rat. Reproductive toxicology (Elmsford, N.Y.) 77, 1-24, https://doi.org/10.1016/j.reprotox.2018.01.007 (2018).

2. Ariyuki, F., Ishihara, H., Higaki, K. \& Yasuda, M. A study of fetal growth retardation in teratological tests: relationship between body weight and ossification of the skeleton in rat fetuses. Teratology 26, 263-267, https://doi.org/10.1002/tera.1420260308 (1982).

3. Smithberg, M. \& Runner, M. N. Teratogenic effects of hypoglycemic treatments in inbred strains of mice. The American journal of anatomy 113, 479-489, https://doi.org/10.1002/aja.1001130308 (1963).

4. Hannah, R. S. \& Moore, K. L. Effects of fasting and insulin on skeletal development in rats. Teratology 4, 135-139, https://doi. org/10.1002/tera.1420040204 (1971).

5. Jensen, V. M. et al. Gestational hypoglycaemia restricts foetal growth and skeletal ossification in the rat. J Preg Child Health 4, https:// doi.org/10.4172/2376-127X.1000321 (2017).

6. Jensen, V. F. H. et al. Importance of gestational hypoglycaemia for foetal malformations and skeletal development in rats. Reproductive Toxicology., https://doi.org/10.1016/j.reprotox.2019.10.003 (2019).

7. Blumbach, K. et al. Combined role of type IX collagen and cartilage oligomeric matrix protein in cartilage matrix assembly: cartilage oligomeric matrix protein counteracts type IX collagen-induced limitation of cartilage collagen fibril growth in mouse chondrocyte cultures. Arthritis and rheumatism 60, 3676-3685, https://doi.org/10.1002/art.24979 (2009).

8. Rani, P. U. et al. Restoration of normal bone development by human homologue of collagen type II (COL2A1) gene in Col2a1 null mice. Developmental dynamics: an official publication of the American Association of Anatomists 214, 26-33, doi:10.1002/(sici) 10970177(199901)214:1<26::aid-dvdy3>3.0.co;2-v (1999).

9. Leighton, M. P. et al. Decreased chondrocyte proliferation and dysregulated apoptosis in the cartilage growth plate are key features of a murine model of epiphyseal dysplasia caused by a matn3 mutation. Human molecular genetics 16, 1728-1741, https://doi. org $/ 10.1093 / \mathrm{hmg} / \mathrm{ddm} 121$ (2007).

10. van der Weyden, L. et al. Functional knockout of the matrilin-3 gene causes premature chondrocyte maturation to hypertrophy and increases bone mineral density and osteoarthritis. The American journal of pathology 169, 515-527, https://doi.org/10.2353/ ajpath.2006.050981 (2006).

11. Pirog-Garcia, K. A. et al. Reduced cell proliferation and increased apoptosis are significant pathological mechanisms in a murine model of mild pseudoachondroplasia resulting from a mutation in the C-terminal domain of COMP. Human molecular genetics 16, 2072-2088, https://doi.org/10.1093/hmg/ddm155 (2007).

12. Blumbach, K., Niehoff, A., Paulsson, M. \& Zaucke, F. Ablation of collagen IX and COMP disrupts epiphyseal cartilage architecture. Matrix biology: journal of the International Society for Matrix Biology 27, 306-318, https://doi.org/10.1016/j.matbio.2007.11.007 (2008).

13. Dreier, R., Opolka, A., Grifka, J., Bruckner, P. \& Grassel, S. Collagen IX-deficiency seriously compromises growth cartilage development in mice. Matrix biology: journal of the International Society for Matrix Biology 27, 319-329, https://doi.org/10.1016/j. matbio.2008.01.006 (2008).

14. Bentovim, L., Amarilio, R. \& Zelzer, E. HIF1alpha is a central regulator of collagen hydroxylation and secretion under hypoxia during bone development. Development (Cambridge, England) 139, 4473-4483, https://doi.org/10.1242/dev.083881 (2012).

15. Zelzer, E. et al. VEGFA is necessary for chondrocyte survival during bone development. Development (Cambridge, England) 131, 2161-2171, https://doi.org/10.1242/dev.01053 (2004).

16. Maes, C. et al. VEGF-independent cell-autonomous functions of HIF-1alpha regulating oxygen consumption in fetal cartilage are critical for chondrocyte survival. Journal of bone and mineral research: the official journal of the American Society for Bone and Mineral Research 27, 596-609, https://doi.org/10.1002/jbmr.1487 (2012).

17. Schipani, E. et al. Hypoxia in cartilage: HIF-1alpha is essential for chondrocyte growth arrest and survival. Genes e. development 15, 2865-2876, https://doi.org/10.1101/gad.934301 (2001). 
18. Rajpurohit, R., Koch, C. J., Tao, Z., Teixeira, C. M. \& Shapiro, I. M. Adaptation of chondrocytes to low oxygen tension: relationship between hypoxia and cellular metabolism. Journal of cellular physiology 168, 424-432, doi:10.1002/(sici)1097-4652(199608)168:2<424::aidjcp21>3.0.co;2-1 (1996).

19. Lin, C., McGough, R., Aswad, B., Block, J. A. \& Terek, R. Hypoxia induces HIF-1alpha and VEGF expression in chondrosarcoma cells and chondrocytes. Journal of orthopaedic research: official publication of the Orthopaedic Research Society 22, 1175-1181, https://doi.org/10.1016/j.orthres.2004.03.002 (2004)

20. Cramer, T., Schipani, E., Johnson, R. S., Swoboda, B. \& Pfander, D. Expression of VEGF isoforms by epiphyseal chondrocytes during low-oxygen tension is HIF-1 alpha dependent. Osteoarthritis and cartilage 12, 433-439, https://doi.org/10.1016/j.joca.2004.02.003 (2004).

21. Murata, M., Yudoh, K. \& Masuko, K. The potential role of vascular endothelial growth factor (VEGF) in cartilage: how the angiogenic factor could be involved in the pathogenesis of osteoarthritis? Osteoarthritis and cartilage 16, 279-286, https://doi. org/10.1016/j.joca.2007.09.003 (2008).

22. Exton, J. H. Regulation of gluconeogenesis by glucocorticoids. Monographs on endocrinology 12, 535-546 (1979).

23. Beauloye, V., Ketelslegers, J. M., Moreau, B. \& Thissen, J. P. Dexamethasone inhibits both growth hormone (GH)-induction of insulin-like growth factor-I (IGF-I) mRNA and GH receptor (GHR) mRNA levels in rat primary cultured hepatocytes. Growth hormone \& IGF research: official journal of the Growth Hormone Research Society and the International IGF Research Society 9 , 205-211, https://doi.org/10.1054/ghir.1999.0110 (1999).

24. Kritsch, K. R., Murali, S., Adamo, M. L. \& Ney, D. M. Dexamethasone decreases serum and liver IGF-I and maintains liver IGF-I mRNA in parenterally fed rats. American journal of physiology. Regulatory, integrative and comparative physiology 282, R528-536, https://doi.org/10.1152/ajpregu.00085.2001 (2002).

25. Brenta, G. Why Can Insulin Resistance Be a Natural Consequence of Thyroid Dysfunction? Journal of Thyroid Research 2011, 152850, https://doi.org/10.4061/2011/152850 (2011).

26. Mark, P. J., Augustus, S., Lewis, J. L., Hewitt, D. P. \& Waddell, B. J. Changes in the placental glucocorticoid barrier during rat pregnancy: impact on placental corticosterone levels and regulation by progesterone. Biology of reproduction 80, 1209-1215, https:// doi.org/10.1095/biolreprod.108.073650 (2009).

27. Smink, J. J. et al. Short-term glucocorticoid treatment of piglets causes changes in growth plate morphology and angiogenesis. Osteoarthritis and cartilage 11, 864-871 (2003).

28. Koedam, J. A., Hoogerbrugge, C. M. \& Van Buul-Offers, S. C. Differential regulation of IGF-binding proteins in rabbit costal chondrocytes by IGF-I and dexamethasone. The Journal of endocrinology 165, 557-567 (2000).

29. Weinstein, R. S. et al. Endogenous glucocorticoids decrease skeletal angiogenesis, vascularity, hydration, and strength in aged mice. Aging cell 9, 147-161, https://doi.org/10.1111/j.1474-9726.2009.00545.x (2010).

30. Weinstein, R. S., Jilka, R. L., Parfitt, A. M. \& Manolagas, S. C. Inhibition of osteoblastogenesis and promotion of apoptosis of osteoblasts and osteocytes by glucocorticoids. Potential mechanisms of their deleterious effects on bone. The Journal of clinical investigation 102, 274-282, https://doi.org/10.1172/jci2799 (1998).

31. Haywood, L. \& Walsh, D. A. Vasculature of the normal and arthritic synovial joint. Histology and histopathology 16, 277-284 (2001).

32. Baron, J., Huang, Z., Oerter, K. E., Bacher, J. D. \& Cutler, G. B. Jr. Dexamethasone acts locally to inhibit longitudinal bone growth in rabbits. Am J. Phy. 263, 489-92 (1992).

33. Silvestrini, G. et al. Evaluation of apoptosis and the glucocorticoid receptor in the cartilage growth plate and metaphyseal bone cells of rats after high-dose treatment with corticosterone. Bone 26, 33-42 (2000).

34. Gafni, R. I. et al. Catch-up growth is associated with delayed senescence of the growth plate in rabbits. Pediatric research 50, 618-623, https://doi.org/10.1203/00006450-200111000-00014 (2001).

35. Gerber, H. P. et al. VEGF couples hypertrophic cartilage remodeling, ossification and angiogenesis during endochondral bone formation. Nat Med 5, 623-628, https://doi.org/10.1038/9467 (1999).

36. Yang, X. et al. Matrilin-3 inhibits chondrocyte hypertrophy as a bone morphogenetic protein-2 antagonist. The Journal of biological chemistry 289, 34768-34779, https://doi.org/10.1074/jbc.M114.583104 (2014).

37. Tchetina, E. V. \& Markova, G. A. Regulation of energy metabolism in the growth plate and osteoarthritic chondrocytes. Rheumatology international 38, 1963-1974, https://doi.org/10.1007/s00296-018-4103-4 (2018).

38. Mobasheri, A. Glucose: an energy currency and structural precursor in articular cartilage and bone with emerging roles as an extracellular signaling molecule and metabolic regulator. Frontiers in endocrinology 3, 153-153, https://doi.org/10.3389/ fendo.2012.00153 (2012).

39. Ryan, H. E., Lo, J. \& Johnson, R. S. HIF-1 alpha is required for solid tumor formation and embryonic vascularization. The EMBO journal 17, 3005-3015, https://doi.org/10.1093/emboj/17.11.3005 (1998).

40. Iyer, N. V. et al. Cellular and developmental control of $\mathrm{O} 2$ homeostasis by hypoxia-inducible factor 1 alpha. Genes \&. development 12, 149-162, https://doi.org/10.1101/gad.12.2.149 (1998).

41. Seagroves, T. N. et al. Transcription factor HIF-1 is a necessary mediator of the pasteur effect in mammalian cells. Molecular and cellular biology 21, 3436-3444, https://doi.org/10.1128/mcb.21.10.3436-3444.2001 (2001).

42. Pereira, R. M., Delany, A. M. \& Canalis, E. Cortisol inhibits the differentiation and apoptosis of osteoblasts in culture. Bone 28, 484-490 (2001)

43. Jia, D., O'Brien, C. A., Stewart, S. A., Manolagas, S. C. \& Weinstein, R. S. Glucocorticoids act directly on osteoclasts to increase their life span and reduce bone density. Endocrinology 147, 5592-5599, https://doi.org/10.1210/en.2006-0459 (2006).

44. Ornoy, A. Transplacental effects of cortisone and vitamin D 2 on the osteogenesis and ossification of fetal long bones in rats. Israel journal of medical sciences $7,540-543$ (1971).

45. EU. Directive 2004/10/EC of the European Parliament and of the Council of 11 February 2004. European Parliament. Official Journal of the European Union. 50, 44-59, http://eur-lex.europa.eu/legal-content/EN/NOT/? uri=CELEX:32004L0010 (2004).

46. ECD. OECD Principles on Good Laboratory Practice. Environment Directorate Paris OECD, ENV/MC/CHEM 17(98), 1-41, OECD series on principles of good laboratory practice and compliance monitoring. http://www.oecd.org/officialdocuments/ publicdisplaydocumentpdf/?cote=ENV/MC/CHEM(98)17\&docLanguage=En (1998).

47. Government of UK. The Good Laboratory Practice (Codification Amendments Etc.) Regulations 2004. UK Statutory Instruments, 944 United Kingdom, 1, 1-1, http://www.legislation.gov.uk/uksi/2004/994/made\#f00004 (2004).

48. EU. Directive 2010/63/EU on the protection of animals used for scientific purposes. The European Parliament, Official Journal of the European Union. 276, 33-79, https://eur-lex.europa.eu/legal-content/EN/TXT/PDF/? (2010).

49. Gundersen, H. J. \& Jensen, E. B. The efficiency of systematic sampling in stereology and its prediction. Journal of microscopy 147, 229-263 (1987)

50. Burdan, F. et al. Morphology and physiology of the epiphyseal growth plate. Folia histochemica et cytobiologica/Polish Academy of Sciences, Polish Histochemical and Cytochemical Society 47, 5-16, https://doi.org/10.2478/v10042-009-0007-1 (2009).

51. Bock, T., Pakkenberg, B. \& Buschard, K. Increased islet volume but unchanged islet number in ob/ob mice. Diabetes 52, 1716-1722 (2003). 


\section{Acknowledgements}

The authors thank Marianne S. Fjording and laboratory technicians Merete H. Nielsen and Sonia R. Araujo (Novo Nordisk A/S) for assistance regarding plasma IGF-1 and corticosterone quantification. We also recognise and appreciate the assistance from laboratory technicians Joan H. Rasmussen, Anette H. Mast, Karsten Marckstrøm, and Line F. Krohn (Novo Nordisk A/S) for immunohistochemic staining and in situ hybridization, as well as Lisa Jansson (Lund University) for technical assistance with qPCR. This work was funded by Novo Nordisk A/S and the STAR Fellowship Programme. Additional support was awarded from the Swedish Research Council (K201552X-14691-13-4), Swedish Research Council for Health, Working Life and Welfare (Grant 2007-2125), Greta and Johan Kock Foundation, Albert Påhlsson Foundation, Alfred Osterlund Foundation, Herman Järnhardt foundation, King Gustav V 80 year fund, Thelma Zoegas Foundation, Swedish Rheumatism foundation, Skåne University Hospital Research Fund and the Research and Development Council of Region Skåne, Sweden. Open access funding provided by Lund University.

\section{Author contributions}

V.F.H.J.: Conceptualization, methodology, formal analysis, investigation, data curation, visualization, supervision, project administration, funding acquisition, and is guarantor. A.M.M.: Conceptualization, methodology, validation, investigation, supervision, project administration. I.B.B.: Conceptualization, methodology, supervision, project administration, funding acquisition. J.N.: Resources, supervision, project administration. B.M.V.: Interpretation. C.L.M.R. and L.P.: Methodology, validation, formal analysis, investigation, visualization. J.J.F. and S.H.M.: Validation, supervision, interpretation. F.E.M.: Data curation, funding acquisition. P.T.N.: Methodology, resources, interpretation, supervision, project administration. J.L.: Resources, supervision, project administration. K.E.A.: Resources, data curation, supervision, project administration, funding acquisition. All authors have approved the submitted version.

\section{Competing interests}

V.F.H.J., A.M.M., I.B.B., J.N., B.M.V., S.H.M. and J.J.F. performed the work as employees of Novo Nordisk A/S. C.L.M.R., L.P., P.T.N., J.L., F.E.M. and K.E.A. declare no potential conflict of interest.

\section{Additional information}

Supplementary information is available for this paper at https://doi.org/10.1038/s41598-020-62554-2.

Correspondence and requests for materials should be addressed to V.F.H.J.

Reprints and permissions information is available at www.nature.com/reprints.

Publisher's note Springer Nature remains neutral with regard to jurisdictional claims in published maps and institutional affiliations.

Open Access This article is licensed under a Creative Commons Attribution 4.0 International License, which permits use, sharing, adaptation, distribution and reproduction in any medium or format, as long as you give appropriate credit to the original author(s) and the source, provide a link to the Creative Commons license, and indicate if changes were made. The images or other third party material in this article are included in the article's Creative Commons license, unless indicated otherwise in a credit line to the material. If material is not included in the article's Creative Commons license and your intended use is not permitted by statutory regulation or exceeds the permitted use, you will need to obtain permission directly from the copyright holder. To view a copy of this license, visit http://creativecommons.org/licenses/by/4.0/.

(C) The Author(s) 2020 